\title{
Molecular Tomography of the Quantum State by Time-Resolved Electron Diffraction
}

\author{
A. A. Ischenko \\ Moscow Lomonosov State University of Fine Chemical Technologies, Vernadskogo prosp. 86, Moscow 119589, Russia
}

Correspondence should be addressed to A. A. Ischenko; aischenko@yasenevo.ru

Received 18 March 2013; Revised 11 July 2013; Accepted 14 October 2013

Academic Editor: Ashok Chatterjee

Copyright (C) 2013 A. A. Ischenko. This is an open access article distributed under the Creative Commons Attribution License, which permits unrestricted use, distribution, and reproduction in any medium, provided the original work is properly cited.

\begin{abstract}
A procedure is described that can be used to reconstruct the quantum state of a molecular ensemble from time-dependent internuclear probability density functions determined by time-resolved electron diffraction. The procedure makes use of established techniques for evaluating the density matrix and the phase-space joint probability density, that is, the Wigner function. A novel expression for describing electron diffraction intensities in terms of the Wigner function is presented. An approximate variant of the method, neglecting the off-diagonal elements of the density matrix, was tested by analyzing gas electron diffraction data for $\mathrm{N}_{2}$ in a Boltzmann distribution and TRED data obtained from the $193 \mathrm{~nm}$ photodissociation of $\mathrm{CS}_{2}$ to carbon monosulfide, CS, at 20, 40, and $120 \mathrm{~ns}$ after irradiation. The coherent changes in the nuclear subsystem by time-resolved electron diffraction method determine the fundamental transition from the standard kinetics to the dynamics of the phase trajectory of the molecule and the tomography of molecular quantum state.
\end{abstract}

\section{Introduction}

In accordance with basic quantum principles, the state of an individual molecule cannot be determined experimentally [1]. However, for an ensemble of similarly prepared systems, it is possible to determine their state operator, the socalled density matrix. Knowing the state of a system means having the maximum possible information about all physical quantities of interest available [2]. The density matrix and the joint phase-space probability density, or Wigner function, $[3,4]$ have a one-to-one correspondence [5] that describes the maximal statistical information available. Thus, in the following text, when the term molecular quantum state is used, we mean the quantum state of an ensemble of similarly prepared molecular species.

In 1933 it was demonstrated by Freenberg [6] (see also $[1$, page 71$])$ that, in principle, a pure quantum state $|\Psi\rangle$ can be reconstructed from the time-dependent coordinate probability density $P(r, t)=|\Psi(r, t)|^{2}$ and its derivative $\partial P(r, t) / \partial t$. It was shown by Weigert [7] that a pure quantum state may also be reconstructed by measuring the distribution $P(r, t)$ at time $t$ and monitoring its evolution after a short time interval $\Delta t$; that is, $P(r, t+\Delta t)=|\Psi(r, t+\Delta t)|^{2}$.
For mixed quantum states, the method of optical homodyne tomography to measure the Wigner function (and density matrix) was first demonstrated by Smithey et al. [8, 9] for both vacuum and squeezed vacuum states of a single spatial-temporal mode in an applied electromagnetic field. (A number of investigations into the preparation and measurement of the quantum state of light may be found in [10].)

In experiments of Kurtsiefer et al. [11, 12], it is demonstrated that Wigner's function can be reconstructed from double-slit experiments involving a coherent beam of helium atoms. Their result $[11,12]$ shows that the joint phase-space probability density $W(r, p)$, where $p$ is the momentum, exhibits negative areas indicating purely nonclassical behavior, such as what might be expected of a particle in an atomic interferometer when it is in a linear superposition of states at two separate locations (see also comments in [13]).

In studies of molecular states, measurements of timedependent fluorescence spectra make it possible to reconstruct Wigner's function for single molecular vibrational modes excited by short optical pulses. This technique is referred to as Molecular Emission Tomography (MET) [14] and was used by Dunn et al. in studies of electronically excited $\mathrm{Na}_{2}$ wave packets, moving in a suggested harmonic potential. 
In the current paper, it is described how time-resolved electron diffraction (TRED) may be used in tomographic studies of the molecular state. TRED [15-17] provides experimental observations having a direct correspondence to the molecular time-dependent probability density function $P(r, t)$. In TRED experiments, a pulsed electron source is used to probe an ensemble of similarly prepared species throughout a sequence in time. The diagnostic electron pulses and the concomitant exciting laser pump pulses are synchronized in such way that stroboscopic diffraction pictures are obtained. In this way, time is introduced as a new variable into the otherwise conventional diffraction technique [15-17]. Thus, reacting laser-excited species, transient states of chemical reactions, or molecular wave packets can be investigated [15-20]. The dynamics of wave packets, as the coherent superposition of quantum states created by short, phasecontrolled optical pulses [21-24], can also be probed. Coherent nuclear dynamics can be followed in this way [15, 25-27] because the elastic scattering of fast electrons proceeds on an ultrashort (ultimately attosecond) time scale and represents a nondestructive probing technique $[15,25,26]$.

\section{Theory}

2.1. General Aspects. TRED experiments yield time-dependent molecular scattering intensity functions, $M(s, t)[15,25$, $26]$, where $|\mathbf{s}|=2|\mathbf{k}| \sin (\theta / 2)$, $\mathbf{k}$ is the momentum vector of the scattered electrons, and $\theta$ is the scattering angle:

$$
M(s, t)=\left(\frac{I_{0}}{R^{2}}\right) g(s) \int P(r, t) \exp (i \mathbf{s r}) d r .
$$

In (1), $I_{0}$ is the incident electron beam profile, $R$ is the scattering distance, $g(s)$ are the scattering functions [28], and $\mathbf{s r}$ is the dot product of the scattering vector $\mathbf{s}$ and internuclear distance vector $\mathbf{r}$. Integration in (1) and in the following equations is over the infinite space if not indicated otherwise.

In classical mechanics, there are no equations that describe the evolution of the probability densities $P(r, t)$ (or $P(p, t)$, where $p$ is momentum); only joint probability densities, $W_{\mathrm{cl}}(p, r, t)$, can be expressed by the Liouville equation. Therefore, corresponding quantum equations for $P(r, t)$, as used in (1), and $P(p, t)$ cannot exist. However, the well known Wigner-Liouville equation [4] can be used to describe the evolution of the Wigner function, $W(p, r, t)$. In order to derive an equation that expresses the timedependent molecular intensities $M(s, t)$ in terms of the Wigner function, the marginal properties of $W(p, r, t)[3,4]$ must be considered. That is,

$$
\begin{aligned}
& \int W(p, r, t) d p=P(r, t), \\
& \int W(p, r, t) d r=P(p, t) .
\end{aligned}
$$

Hence, (1) can be expressed as follows:

$$
M(s, t)=\left(\frac{I_{0}}{R^{2}}\right) g(s) \int d p \int W(p, r, t) \exp (i \mathbf{s r}) d r .
$$

Equation (4) is the most general representation of TRED molecular intensities in terms of the Wigner function. In this representation, $M(s, t)$ can be interpreted as the filtered projection of the Wigner function, with the scattering operator $\exp (i$ sr) as the filter, modified by the scattering functions $g(s)$. For a number of problems, $W(p, r, t)$ can be evaluated analytically (see, e.g., the review in [4]) or by solving the Wigner-Liouville equation numerically with appropriate molecular potential functions.

In (1) and (2), $P(r, t)=|\Psi(r, t)|^{2}$. The wave function $\Psi(r, t)$ can be expanded in terms of the orthonormal basis functions $\psi_{n}(r)$ in the following way $[1,2]$ :

$$
\Psi(r, t)=\sum_{n} C_{n} \psi_{n}(r) \exp \left(-i \omega_{n} t\right)
$$

where $n$ is the quantum number of the state energy $E_{n}, \omega_{n}$ is the oscillator frequency, and $C_{n}$ is the amplitude. Then, (1). can be written as

$$
\begin{aligned}
M(s, t)= & \left(\frac{I_{0}}{R^{2}}\right) g(s) \\
& \times \sum_{m, n} \boldsymbol{\rho}_{m n} \exp \left(i\left(\omega_{m}-\omega_{n}\right) t\right) \\
& \times\left\langle\psi_{m}(r)|\exp (i \mathbf{s r})| \psi_{n}(r)\right\rangle,
\end{aligned}
$$

where the $\boldsymbol{\rho}_{m n}$ are the density matrix elements.

From (6) it is seen that UED molecular intensities depend explicitly on the quantum state of a molecular system. Likewise, the $P(r, t)$ obtained from TRED data, that is, the Fourier transform of (1),

$$
P(r, t)=\left(\frac{2}{\pi}\right)^{1 / 2}\left(\frac{R^{2}}{I_{0}}\right) \int M(s, t)[g(s)]^{-1} \exp (- \text { isr }) d s
$$

depends on both the position $r$ and the time $t$ and contains implicitly all information on the quantum state of the system, being a projection or "shadow" [29] of the Wigner function. A simple sequence of TRED measurements of the position-dependent probability density provides the necessary information for tomographic reconstruction of the Wigner function. For this purpose, the inverse Radon transformation can be applied [29, 30].

Another possibility for molecular quantum state reconstruction is the evaluation of the density matrix elements, $\boldsymbol{\rho}_{m n}$, which have a one-to-one correspondence [5] to Wigner's function:

$$
W(r, p)=\left(\frac{1}{\pi \hbar}\right) \int \exp \left(\frac{2 i p x}{\hbar}\right)\langle r-x|\rho| r+x\rangle d x,
$$

where $\rho$ is the density matrix [2]:

$$
\boldsymbol{\rho}=\sum_{n, m} \boldsymbol{\rho}_{m n}\left|\psi_{n}(r)\right\rangle\left\langle\psi_{m}(r)\right|
$$

2.2. Approximate Procedures. When the TRED measurements do not span a complete cycle and the data are incomplete, only the diagonal density matrix elements, $\boldsymbol{\rho}_{n}=\boldsymbol{\rho}_{n n}$, 
can be evaluated from the probability density $\langle P(r, t)\rangle_{\tau}$ averaged over the time interval $\tau \gg \sup \left(\left|\omega_{m}-\omega_{n}\right|^{-1}\right)$, with $m \neq n$, as shown by Richter and Wünsche [31-34], Leonhardt $[29,35]$, Leonhardt and Raymer [36], and others [37-39]. This method uses the superposition principle combined with the structure of Schrödinger's equation to reveal the quantum state of a molecule from the time-averaged coordinatedependent probability density function. In general, and for a complete data set,

$$
\boldsymbol{\rho}_{m n}=\int P\left(r, \omega_{m}-\omega_{n}\right)\left\{\frac{\partial\left[\psi_{m}(r) \varphi_{n}(r)\right]}{\partial r}\right\} d r,
$$

where $P\left(r, \omega_{m}-\omega_{n}\right)$ is the Fourier transform of the timedependent probability density $P(r, t)$ [29, equation 5.59], the $\psi_{n}(r)$ are the normalized regular solutions of the timeindependent Schrödinger equation, and the $\varphi_{n}(r)$ are the linearly independent nonnormalizable irregular second solutions $[33,36,40]$.

In the particular case of incomplete data, one can express the diagonal density matrix elements as

$$
\boldsymbol{\rho}_{n n}=\int\langle P(r, t)\rangle_{\tau}\left\{\frac{\partial\left[\psi_{n}(r) \varphi_{n}(r)\right]}{\partial r}\right\} d r .
$$

Time-dependent probability density function $P(r, t)$ averaged over electron pulse profile of duration $\tau$ provides evolution in time of the diagonal density matrix elements $\boldsymbol{\rho}_{n n}$ in (11).

The terms state, density matrix, or Wigner function are, for most practical purposes, synonymous. Thus, when the available data are incomplete and only the diagonal density matrix elements are used, one has to keep in mind that it is not possible to determine the complete Wigner function. However, it is possible to use the diagonal density matrix elements to calculate an approximate or truncated Wigner function, which contains useful information when the offdiagonal elements are small. The truncated Wigner functions can be used to calculate approximate von Neumann entropies and purity parameters which, nonetheless, may contain meaningful information on the quantum state of a system.

\section{Experiment}

Instrumentation for TRED developed at the University of Arkansas, spanning the time domain from microseconds to picoseconds, has been described before [41]. A UV laserdriven tantalum photocathode in a Pierce-type [42] electron gun assembly was used for generation of the probe electron pulses. The mechanics of the photoemission process suggest that the electron pulse should follow the temporal profile of the incident optical pulse $[43,44]$. In the experiments used for this study, the output from an excimer laser (Questek Model 2260, $193 \mathrm{~nm}$ (ArF), pulse duration 15 ns) was used to generate electron pulses (duration $\sim 15 \mathrm{~ns}$ FWHM, $\sim 10^{10}$ electrons per pulse [41]) which were accelerated through a potential of $\sim 40 \mathrm{keV}$. For the current study, carbon disulfide, $\mathrm{CS}_{2}$, target molecules were irradiated with similar $193 \mathrm{~nm}$ laser pulses from a second excimer at 50-130 mJ per pulse.

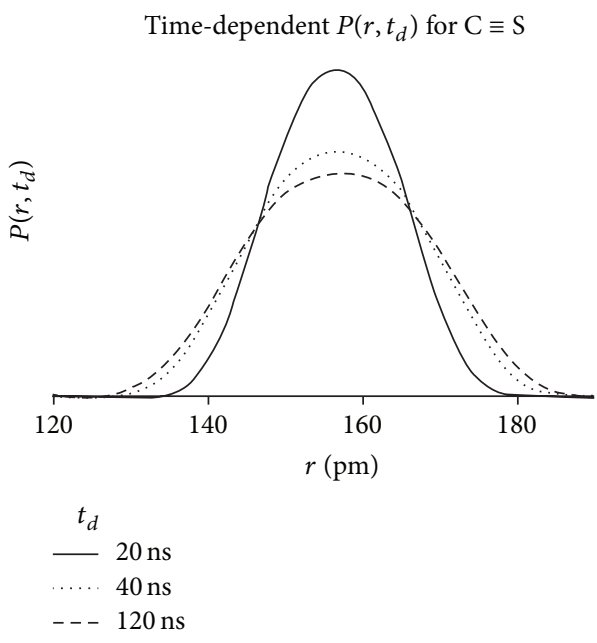

(a)

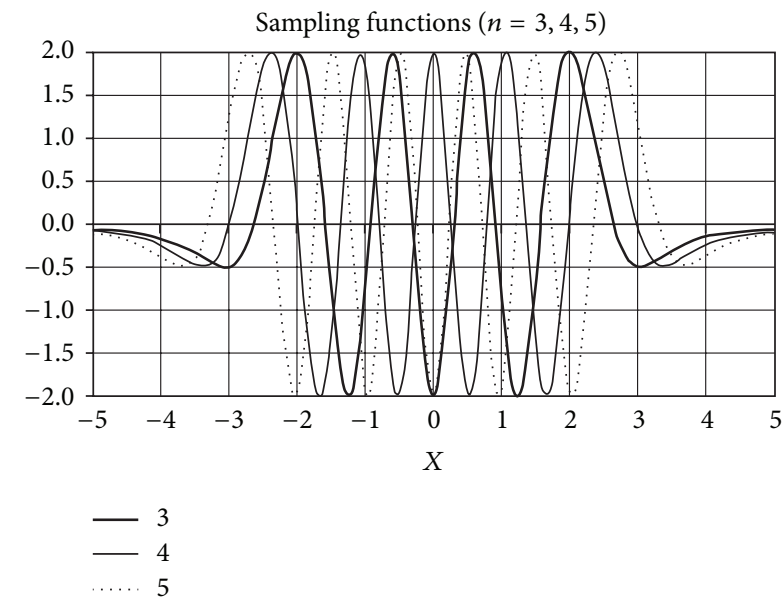

(b)

FIGURE 1: Experimental probability densities for CS at 20, 40, and $120 \mathrm{~ns}$ following irradiation of $\mathrm{CS}_{2}$ at $193 \mathrm{~nm}$, as calculated from the stochastic approach [45], and selected sampling functions (lower curves). The sampling procedure was applied for $n<10$ and the dimensionless variable $x=\left(2 \pi \mu \omega_{e} / h\right)^{1 / 2}\left(r-r_{e}\right)$, where $\mu$ is the reduced mass, $\omega_{e}$ the harmonic frequency, and $r_{e}$ the equilibrium internuclear distance. Sampling functions for CS (bottom) are defined in (14).

Delay times between excitation and electron scattering varied from 20 to $120 \mathrm{~ns}$ and the diffraction intensities were recorded online using a phosphor screen-photodiode array detector [41]. The target gases were admitted into the vacuum chamber through a nozzle orifice (I.D. $200 \mu \mathrm{m}$ ) at a pushing pressure of 30-50 Torr. The electron scattering occurred in a volume immediately adjacent to the nozzle exit in which the gas pressure is estimated to be $\sim 10$ Torr. Such conditions preclude the formation of clusters, and the number $Z$ of collisions per particle in the target gas is estimated to be in the range of $10^{0}<Z<10^{2}$ in $15 \mathrm{~ns}$. Also, time averaging is determined by the electron pulse profile and averaging time $\tau \sim 15 \mathrm{~ns}$. The experimental TRED data sets [45] and sampling functions are presented in Figure 1. 
TABLE 1: Diagonal density matrix elements for $\mathrm{N}_{2}$ and CS.

\begin{tabular}{|c|c|c|c|c|c|c|c|c|c|c|c|c|c|c|c|}
\hline$n / \boldsymbol{\rho}_{n n}$ & 0 & 1 & 2 & 3 & 4 & 5 & 6 & 7 & 8 & 9 & 10 & 11 & 12 & 13 & 14 \\
\hline $\mathrm{N}_{2}(\exp )^{\mathrm{a}}$ & 0.9938 & 0.0011 & 0.0102 & -0.000 & 0.001 & 0.005 & -0.009 & 0.012 & -0.011 & 0.009 & -0.005 & - & - & - & - \\
\hline $\mathrm{N}_{2}$ (theor) $^{\mathrm{b}}$ & 0.9935 & 0.0064 & 0.0000 & 0.000 & 0.000 & 0.000 & 0.000 & 0.000 & 0.000 & 0.000 & 0.000 & 0.000 & 0.000 & 0.000 & 0.000 \\
\hline $\mathrm{CS}(\text { nasc })^{\mathrm{c}}$ & 0.000 & 0.049 & 0.098 & 0.156 & 0.101 & 0.077 & 0.061 & 0.070 & 0.073 & 0.077 & 0.077 & 0.073 & 0.067 & 0.021 & 0.000 \\
\hline $\mathrm{CS}\left(\right.$ nasc) ${ }^{\mathrm{d}}$ & 0.000 & 0.074 & 0.148 & 0.236 & 0.153 & 0.116 & 0.083 & 0.042 & 0.042 & 0.032 & 0.023 & 0.023 & 0.028 & - & - \\
\hline $\operatorname{CS}(20 n s)^{e}$ & 0.349 & 0.260 & 0.172 & 0.099 & 0.053 & 0.025 & 0.013 & 0.000 & -0.001 & -0.003 & 0.001 & - & - & - & - \\
\hline $\mathrm{CS}(40 \mathrm{~ns})^{\mathrm{e}}$ & 0.204 & 0.190 & 0.160 & 0.129 & 0.097 & 0.075 & 0.058 & 0.033 & 0.005 & -0.015 & -0.015 & - & - & - & - \\
\hline CS $(120 \mathrm{~ns})^{\mathrm{e}}$ & 0.173 & 0.165 & 0.147 & 0.124 & 0.100 & 0.080 & 0.058 & 0.040 & 0.029 & 0.021 & 0.013 & - & - & - & - \\
\hline
\end{tabular}

${ }^{a}$ Values obtained from experimental electron diffraction intensities of $\mathrm{N}_{2}$ in an equilibrium Boltzmann distribution recorded at $673 \mathrm{~K}$. Estimated uncertainties are 0.01. ${ }^{\mathrm{b}}$ Values calculated for a Boltzmann distribution at $T=673 \mathrm{~K} .{ }^{\mathrm{c}}$ Values derived from the nascent ensemble of CS reported in [46]. ${ }^{\mathrm{d}}$ Values derived from the nascent ensemble of CS reported in [47]. ${ }^{\mathrm{e}}$ Derived from TRED data of photogenerated CS recorded at 20,40, and $120 \mathrm{~ns}$ after the $193 \mathrm{~nm}$ photodissociation of $\mathrm{CS}_{2}$.

To further illustrate the described procedures, $\mathrm{N}_{2}$ in an equilibrium Boltzmann distribution was chosen as a test case because its potential energy function is well known from high-resolution spectroscopic measurements. Data were recorded throughout the temperature range from $298 \mathrm{~K}$ to $773 \mathrm{~K}$, with a continuous electron beam [15, 41]. Experimental data and sampling functions are presented in Figure 2.

\section{Results and Discussion}

The photochemistry of $\mathrm{CS}_{2}$ has been thoroughly studied in the absorption region from 180 to $210 \mathrm{~nm}$ [45-47]. At $193 \mathrm{~nm}$, and in single-photon processes, the photodissociation proceeds according to the following two-channel scheme:

$$
\begin{gathered}
\mathrm{CS}_{2}\left(X^{1} \Sigma_{g}^{+}\right) \longrightarrow \mathrm{CS}_{2}\left({ }^{1} B_{2}{ }^{1} \Sigma_{u}^{-}\right) \longrightarrow \operatorname{CS}\left(X^{1} \Sigma_{g}^{+}\right)+S\left({ }^{3} P_{J}\right) \\
\mathrm{CS}_{2}\left(X^{1} \Sigma_{g}^{+}\right) \longrightarrow \mathrm{CS}_{2}\left({ }^{1} B_{2}{ }^{1} \Sigma_{u}^{-}\right) \longrightarrow \operatorname{CS}\left(X^{1} \Sigma_{g}^{+}\right)+S\left({ }^{1} D_{J}\right) .
\end{gathered}
$$

The structure of excited state of $\mathrm{CS}_{2}$ is believed to be quasilinear, but with different geometrical parameters compared to the ground state; further, predissociation occurs on a time scale of $\sim 1$ ps.

In supersonic molecular beams of $193 \mathrm{~nm}$ irradiated $\mathrm{CS}_{2}$, it was found $[46,47]$ that the nascent vibrational distribution of CS is strongly inverted for both the ${ }^{1} D_{2}$ and ${ }^{3} P_{2}$ dissociation channels, exhibiting a bimodal [46] or broad [47] distribution with low occupancy at $n=0$ and $n \geq 10$, as summarized in Table 1.

To apply the quantum sampling procedure to $\mathrm{N}_{2}$ as a test equilibrium case, position-probability densities were first derived from the diffraction intensities by using the stochastic approach to data analysis [45]. This approach [45] allows one to refine TRED data of laser-excited species without imposing any model restrictions on intramolecular motion. It is an advantage of the procedure that the requisite molecular parameters may be refined from the experimental data without any information on the potential energy surface.

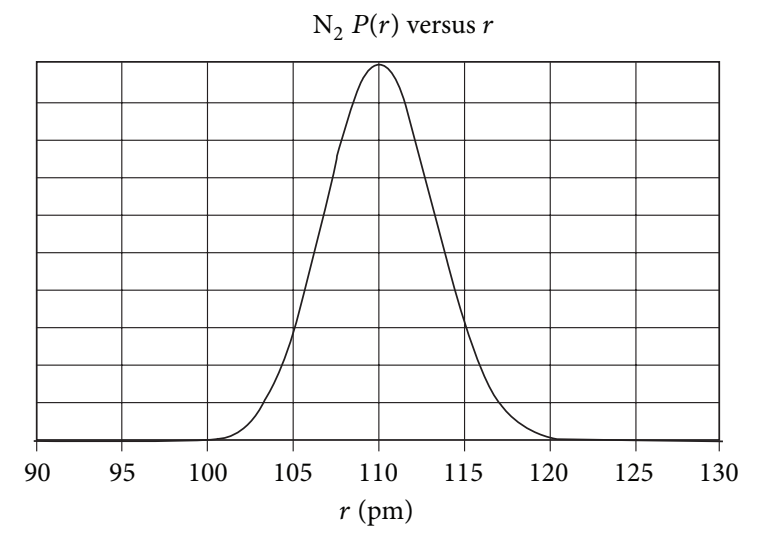

(a)

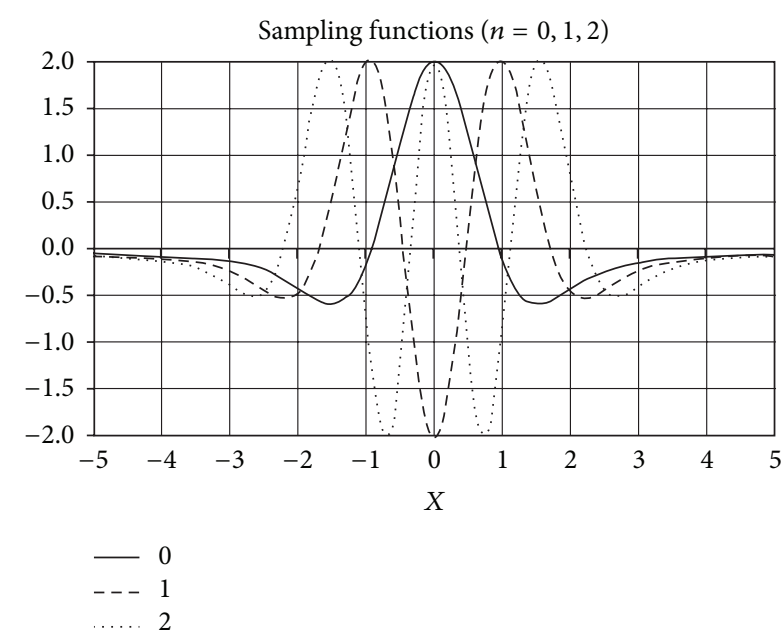

(b)

FIGURE 2: Experimental probability density for $\mathrm{N}_{2}$ (upper curve) in an equilibrium distribution at $673 \mathrm{~K}$; note the slight asymmetry of the $P(r)$ due to the anharmlonicity of the molecular vibration. Typical sampling functions (lower curves) selected for illustration. The sampling procedure was applied for $n<10$ and the dimensionless variable $x=\left(2 \pi \mu \omega_{e} / h\right)^{1 / 2}\left(r-r_{e}\right)$, where $\mu$ is the reduced mass, $\omega_{e}$ is the harmonic frequency, and $r_{e}$ is the equilibrium internuclear distance. Sampling functions for $\mathrm{N}_{2}$ (bottom) are defined in (14). 


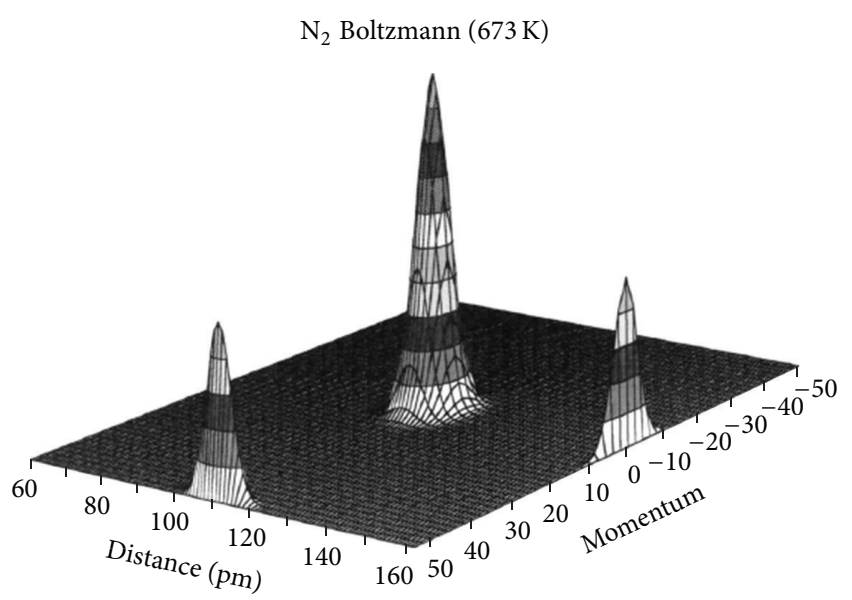

Figure 3: Wigner function for $\mathrm{N}_{2}$ in a Boltzmann distribution reconstructed from the experimental electron diffraction results of $[41,45]$. The marginal $P(r)$ and $P(p)$ at the left and right edges of the graph, respectively, are plotted on an arbitrary vertical scale; momentum scale is in arbitrary units. For this equilibrium case, the diagonal density matrix elements and Wigner function adequately describe the full quantum state of the system.

The probability density functions obtained in this way were then sampled with the functions $F_{m n}(r)$ [34]:

$$
F_{m n}(r)=\frac{\partial\left[\psi_{m}(r) \varphi_{n}(r)\right]}{\partial r},
$$

where $\psi_{m}(r)$ and $\varphi_{n}(r)$ are, respectively, the normalized regular and nonnormalizable irregular wave functions referred to above. The sampling functions for the first 3 levels are shown in Figure 2. Calculated density matrix elements for the Boltzmann distribution of $\mathrm{N}_{2}$ at $T=673 \mathrm{~K}$ are compared with the experimentally determined values in Table 1.

The truncated Wigner function, together with the associated marginal position- and momentum-dependent probability density functions ((2) and (3)), is shown in Figure 3. The results represent, for the nearly harmonic onedimensional oscillator at equilibrium, a Gaussian-like function and Gaussian-like marginal position- and momentumprobability densities.

For CS, we derived time-dependent probability density functions $\langle P(r, t)\rangle_{\tau}=P\left(r, t_{d}\right)$ at several delay times from the experimental time-dependent molecular intensity functions, using the stochastic approach $[15,45]$. To calculate the sampling functions of (14), the Dunham vibrational potential of CS, as determined spectroscopically up to sixth order [48], was used. The regular wave functions $\psi_{n}(r)$ were evaluated by solving Schrödinger's equation for the same Dunham potential [48] using the variational technique of [49].

The values of the diagonal density matrix elements, $\boldsymbol{\rho}_{n n}$, obtained by applying (11) to the experimental $P\left(r, t_{d}\right)$ at different delay times, are represented in Table 1 . Truncated Wigner functions for $t_{d}=20 \mathrm{~ns}, 40 \mathrm{~ns}$, and $120 \mathrm{~ns}$ are shown in Figure 4 and compared with those for the nascent state distribution of [47] (see also Table 1). For $t_{d}=40$ and 120 ns the truncated Wigner functions and the corresponding marginal density functions, $P(r, t)$ and $P(p, t)((2)$ and $(3))$, are similar. In contrast, these functions exhibit significant changes between $t_{d}=0,20$, and $40 \mathrm{~ns}$. We interpret these changes in the following way.

Changes in the first 20 ns of the evolution of the system are indicative of rapid equilibration, predominantly by noncollisional intermolecular vibrational energy transfer involving, for example, dipole-dipole interactions. During this time span bimodal distributions, both in position and momentum space, evolve to monomodal and narrower distributions. Between 20 and 40 ns, collision-induced energy transfer becomes more important than collisionless energy transfer processes. Thus, we believe that the changes between 20 and $40 \mathrm{~ns}$ indicate electronic to vibrational energy transfer induced by collisions between $S\left({ }^{1} D_{J}\right)$ atoms and $\operatorname{CS}\left(X^{1} \Sigma_{g}^{+}\right)$ molecules. Between 40 and 120 ns, this process shows features of saturation.

It can be inferred from Table 1 that, keeping the foregoing approximations in mind, there is significant depopulation of the first three vibrational levels of CS between 20 and $120 \mathrm{~ns}$, very likely indicating the transfer of vibrational energy to rotational and translational degrees of freedom. This interpretation is supported by the fact that the rotational temperature of CS in this process increases from $T_{\text {rot }}=4200 \pm$ $300 \mathrm{~K}$ at $20 \mathrm{~ns}$ to $5000 \pm 600 \mathrm{~K}$ at $40 \mathrm{~ns}$ and to $5200 \pm 800 \mathrm{~K}$ at $120 \mathrm{~ns}$ [45]. The truncated Wigner functions at 20, 40, and $120 \mathrm{~ns}$ are positive throughout the entire phase space, in agreement with the hypothesis that the molecular quantum state of CS after $20 \mathrm{~ns}$ can be characterized as a classicallike statistical mixture, rather than a coherently prepared superposition of the lower vibrational states. This confirms the increasing importance of collisions after $20 \mathrm{~ns}$ and is in contrast to the nascent molecular distribution, for which the complete Wigner function exhibits significant negative areas (not visible in Figure 4). Furthermore in this case, the bimodality of the marginal distributions, $P(r, t)$ and $P(p, t)$, reflects the fact that the $193 \mathrm{~nm}$ photodissociation of $\mathrm{CS}_{2}$ proceeds via two different channels, producing $S\left({ }^{3} P_{J}\right)$ and $S\left({ }^{1} D_{J}\right)$. The symmetric nature of the bimodality shown in Figure 4 is an artifact due to the truncation of the Wigner function.

We have also investigated the processes described above in terms of the von Neumann entropy $S$ [29]:

$$
S=-\operatorname{tr}\{\rho \ln \rho\},
$$

where $\operatorname{tr}\{Q\}$ is the trace of the operator $Q$ and $\rho$ is the density matrix. The von Neumann entropy vanishes for pure states, exceeds zero for mixed states, and is an extensive quantity for nonentangled subsystems; thus, it is a fundamental measure of the preparation impurity of a quantum state [29]. It is also informative to consider the so-called purity parameter, $S^{\text {pur }}$, which represents a lower bound for the von Neumann entropy; that is, $S^{\text {pur }} \leq S$ :

$$
S^{\text {pur }}=1-\operatorname{tr}\left\{\boldsymbol{\rho}^{2}\right\}=1-2 \pi \hbar \iint[W(p, r, t)]^{2} d p d r .
$$

In the current investigation, the diagonal density matrix elements were used to derive approximate estimates for $S$ 


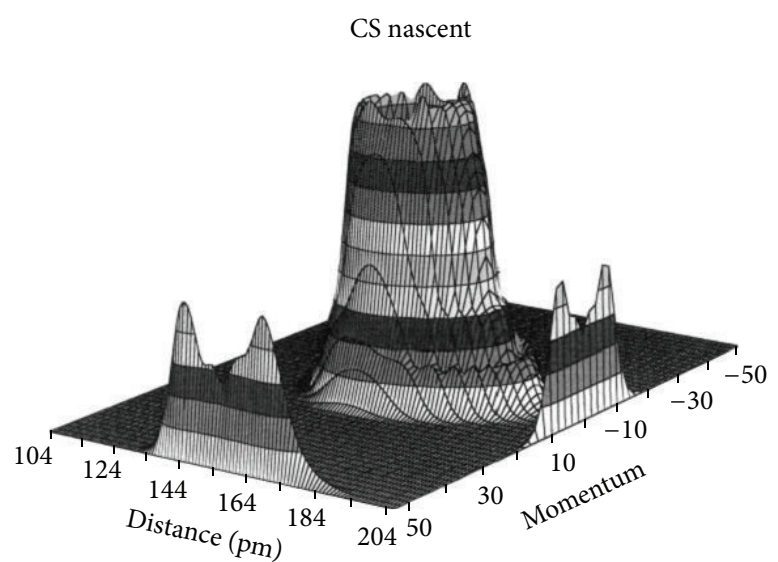

(a)

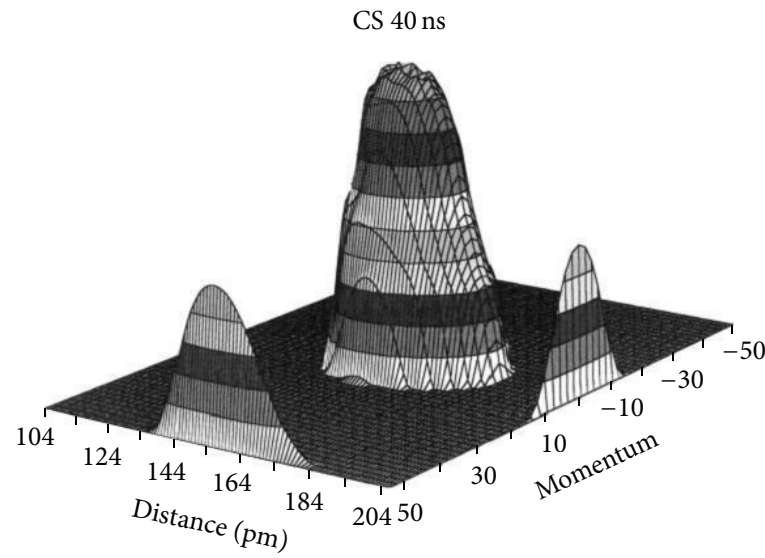

(c)

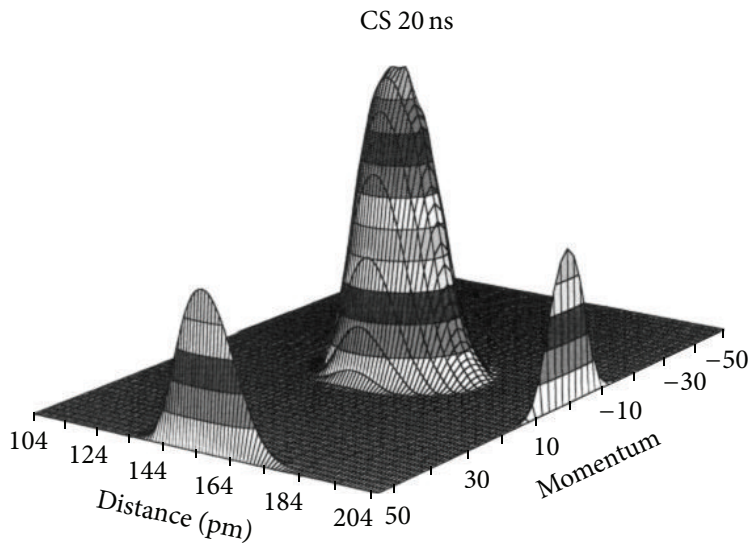

(b)

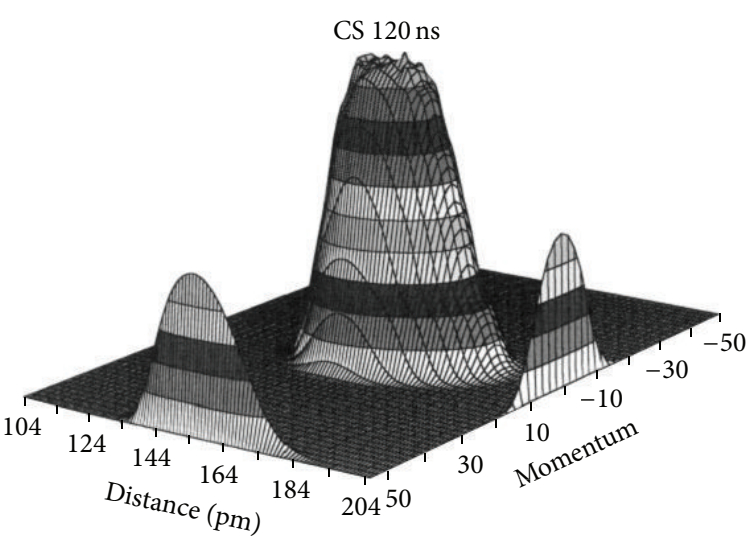

(d)

FIGURE 4: Wigner functions for CS in a nascent distribution (upper left), and at 20, 40, and $120 \mathrm{~ns}$ following photodissociation of CS 2 by $193 \mathrm{~nm}$ laser pulses. For the nascent distribution, the diagonal density matrix was obtained from the time-of-flight measurements of [47]; negative areas are present which are not visible from the chosen perspective. The experimental time-dependent Wigner functions were calculated using the diagonal density matrix elements of Table 1. The marginal probability density functions are shown at the left and right sides of each graph; scales are arbitrary.

and $S^{\text {pur }}$. In spite of their approximate nature, it is seen from Table 2 that the estimates obtained are in agreement with previous descriptions [45] of the $193 \mathrm{~nm}$ photodissociation of $\mathrm{CS}_{2}$. That is, at $20 \mathrm{~ns}$ both the approximated von Neumann entropy and purity parameters are smaller than at $t=0$ (nascent CS), indicating the transition from bimodal to monomodal distributions. At times later than $20 \mathrm{~ns}$, both of these parameters increase due to increasing populations of vibrational states above $n=3$.

\section{Conclusion}

The material presented above shows that time-resolved electron diffraction measurements offer a new avenue for quantum state reconstruction, yielding the Wigner function from a tomographically complete set of time-dependent positionprobability densities. When the TRED data are incomplete, a truncated Wigner function can be determined from the diagonal density matrix elements derived from the timeaveraged experimental probability density $\langle P(r, t)\rangle$. Even though the diagonal terms do not describe the full quantum state of a system, they still contain useful information.

Deriving the truncated Wigner function for $\mathrm{N}_{2}$ in an equilibrium Boltzmann distribution, the expected Gaussianlike features were found. In the case of the $193 \mathrm{~nm}$ photodissociation of $\mathrm{CS}_{2}$, the analysis indicates collisionless vibrational energy transfer mechanisms in the photogenerated ensemble of nascent CS and a collision-induced electronic to vibrational energy transfer process, from $S\left({ }^{1} D\right)$ to $\operatorname{CS}\left(X^{1} \Sigma_{g}^{+}\right)$ at later times. Apart from quantum state reconstruction, (4) represents a novel expression for analyzing diffraction intensities directly in terms of the Wigner function.

Molecular quantum state measurements by TRED are complementary to quantum state preparation and control of matter by laser fields. Coherent nuclear dynamics can be followed in this way [15, 25-27] because the elastic scattering of fast electrons proceeds on an ultrashort (ultimately attosecond) time scale and represents a nondestructive probing technique. Adequately, new avenues in physical chemistry 
TABle 2: Sum of the diagonal density matrix elements $\left(\sum_{n} \boldsymbol{\rho}_{n}\right)$, approximate von Neuman entropies $(S)$, and approximate purity parameters $\left(S^{\text {pur }}\right)$ for $\mathrm{N}_{2}$ and CS.

\begin{tabular}{|c|c|c|c|}
\hline & $\sum_{n} \boldsymbol{\rho}_{n}$ & $S$ & $S^{\text {pur }}$ \\
\hline $\mathrm{N}_{2}(\exp )^{\mathrm{a}}$ & 1.03 & 0.19 & 0.012 \\
\hline $\mathrm{N}_{2}$ (theor) $^{\mathrm{b}}$ & 1.00 & 0.039 & 0.013 \\
\hline $\mathrm{CS}(\text { nasc })^{\mathrm{c}}$ & 1.00 & 2.49 & 0.91 \\
\hline $\mathrm{CS}\left(\right.$ nasc) ${ }^{\mathrm{d}}$ & 1.00 & 2.21 & 0.87 \\
\hline $\operatorname{CS}(20 n s)^{e}$ & 0.97 & 1.56 & 0.77 \\
\hline $\mathrm{CS}(40 \mathrm{~ns})^{\mathrm{e}}$ & 0.95 & 1.92 & 0.86 \\
\hline $\mathrm{CS}(120 \mathrm{~ns})^{\mathrm{e}}$ & 0.90 & 2.11 & 0.88 \\
\hline
\end{tabular}

${ }^{\mathrm{a}}$ Values obtained from experimental electron diffraction intensities of $\mathrm{N}_{2}$ in an equilibrium Boltzmann distribution recorded at $673 \mathrm{~K}$. Estimated uncertainties are $0.01 .{ }^{\mathrm{b}}$ Values calculated for a Boltzmann distribution at $T=673 \mathrm{~K}$. ${ }^{c}$ Values derived from the nascent ensemble of CS reported in [46]. ${ }^{\mathrm{d}}$ Values derived from the nascent ensemble of CS reported in [47]. ${ }^{\mathrm{e}}$ Values derived from TRED data of photogenerated CS recorded at 20, 40, and $120 \mathrm{~ns}$ after the $193 \mathrm{~nm}$ photodissociation of $\mathrm{CS}_{2}$.

are in development based on studies of coherent nuclear dynamics $[15,16,50]$.

Since the 1980, the scientific world made intensive efforts in order to register a movie about the coherent nuclei dynamics in the molecules, the fast dynamic processes in biological tissues and cells, and the structure dynamics of the solid in nanovolumes in time. The observed coherent changes in the nuclear subsystem by time-resolved electron diffraction method determine the fundamental transition from the standard kinetics to the dynamics of the phase trajectory of the molecule and the tomography of molecular quantum state.

\section{Acknowledgments}

Academician of the RAS, A. L. Buchachenko, Professor G. V. Fetisov (Chemistry Department of Moscow Lomonosov State University), Professor L. Schafer (University of Arkansas at Fayetteville, USA), Professor E. A. Ryabov (Institute of Spectroscopy RAS), and Professor V. N. Bagratashvili (IPLIT RAS) are greatly thanked for their helpful discussions. This work was supported by RFBR Grants 12-02-00840-a and 1202-12048-ofi_m.

\section{References}

[1] E. C. Kemble, The Fundamental Principles of Quantum Mechanics, McGraw-Hill, London, UK, 1937.

[2] L. E. Ballentine, Quantum Mechanics, Prentice Hall, Englewood Cliffs, NJ, USA, 1990.

[3] E. P. Wigner, Perspectives in Quantum Theory, edited by W. Yorgrau and A. van der Merve, Dover, New York, NY, USA, 1979.

[4] M. Hillery, R. F. O'Connell, M. O. Scully, and E. P. Wigner, "Distribution functions in physics: fundamentals," Physics Reports, vol. 106, no. 3, pp. 121-167, 1984.

[5] K. E. Cahill and R. J. Glauber, "Density operators and quasiprobability distributions," Physical Review, vol. 177, no. 5, pp. 1882-1902, 1969.
[6] E. Freenberg, The scattering of slow electrons in neutral atoms [Ph.D. thesis], Harvard University, 1933.

[7] S. Weigert, "How to determine a quantum state by measurements: the Pauli problem for a particle with arbitrary potential," Physical Review A, vol. 53, pp. 2078-2083, 1996.

[8] D. T. Smithey, M. Beck, M. G. Raymer, and A. Faridani, "Measurement of the Wigner distribution and the density matrix of a light mode using optical homodyne tomography: application to squeezed states and the vacuum," Physical Review Letters, vol. 70, no. 9, pp. 1244-1247, 1993.

[9] D. T. Smithey, M. Beck, J. Cooper, and M. G. Raymer, "Complete experimental characterization of the quantum state of a light mode via the Wigner function and the density matrix: application to quantum phase distributions of vacuum and squeezedvacuum states," Physica Scripta, vol. 48, p. 35, 1993.

[10] W. P. Schleich and M. G. Raymer, "Quantum state preparation and measurement," Journal of Modern Optics, vol. 44, no. 11-12, 1997.

[11] C. Kurtsiefer, T. Pfau, and J. Mlynek, "Measurement of the wigner function of an ensemble of helium atoms," Nature, vol. 386, no. 6621, pp. 150-153, 1997.

[12] T. Pfau and C. Kurtsiefer, "Partial reconstruction of the motional Wigner function of an ensemble of helium atoms," Journal of Modern Optics, vol. 44, no. 11-12, pp. 2551-2564, 1997.

[13] M. Freyberger and W. P. Schleich, "True vision of a quantum state," Nature, vol. 386, no. 6621, pp. 121-122, 1997.

[14] T. J. Dunn, I. A. Walmsley, and S. Mukamel, "Experimental determination of the quantum-mechanical state of a molecular vibrational mode using fluorescence tomography," Physical Review Letters, vol. 74, no. 6, pp. 884-887, 1995.

[15] A. A. Ischenko, G. V. Girichev, and I. Y. Tarasov, "Electron Diffraction: structure and dynamics of free molecules and condensed matter," in Proceedings of the Femtosecond Electron Imaging and Spectroscopy, Fizmatlit, 2013.

[16] R. Srinivasan, V. A. Lobastov, C.-Y. Ruan, and A. H. Zewail, "Ultrafast electron diffraction (UED): a new development for the $4 \mathrm{D}$ determination of transient molecular structures," Helvetica Chimica Acta, vol. 86, no. 6, pp. 1763-1838, 2003.

[17] W. E. King, G. H. Campbell, A. Frank et al., "Ultrafast electron microscopy in materials science, biology, and chemistry," Journal of Applied Physics, vol. 97, no. 11, pp. 1-27, 2005.

[18] J. R. Dwyer, C. T. Hebeisen, R. Ernstorfer et al., "Femtosecond electron diffraction: 'making the molecular movie," Philosophical Transactions of the Royal Society A, vol. 364, no. 1840, pp. 741-778, 2006.

[19] G. Sciaini and R. J. D. Miller, "Femtosecond electron diffraction: heralding the era of atomically resolved dynamics," Reports on Progress in Physics, vol. 74, Article ID 09610, 2011.

[20] C.-Y. Ruan, Y. Murooka, R. K. Raman, R. A. Murdick, R. J. Worhatch, and A. Pell, "The development and applications of ultrafast electron nanocrystallography," Microscopy and Microanalysis, vol. 15, no. 4, pp. 323-337, 2009.

[21] D. J. Tannor and S. A. Rice, "Control of selectivity of chemical reaction via control of wave packet evolution," The Journal of Chemical Physics, vol. 83, no. 10, pp. 5013-5018, 1985.

[22] J. K. Krause, R. M. Whitnell, K. R. Wilson, and Y. J. Yan, Femtosecond Chemistry, edited by J. Manz and L. Woste, Springer, Weinheim, Germany, 1995.

[23] Y. J. Yan and K. R. Wilson, "Optimal control of molecular dynamics via two-photon processes," Journal of Chemical Physics, vol. 100, p. 1094, 1994. 
[24] J. L. Krause, K. J. Schäfer, M. Ben-Nun, and K. R. Wilson, "Creating and detecting shaped rydberg wave packets," Physical Review Letters, vol. 79, pp. 4978-4981, 1997.

[25] H. Ihee, V. A. Lobastov, U. M. Gomez et al., "Direct imaging of transient molecular structures with ultrafast diffraction," Science, vol. 291, no. 5503, pp. 458-462, 2001.

[26] A. H. Zewail and J. M. Thomas, 4D Electron Microscopy. Imaging in Space and Time, Imperial College Press, London, UK, 2010.

[27] J. C. Williamson and A. H. Zewail, "Ultrafast electron diffraction. 4. Molecular structures and coherent dynamics," Journal of Physical Chemistry, vol. 98, no. 11, pp. 2766-2781, 1994.

[28] R. A. Bonham and M. Fink, High Energy Electron Scattering, Van Nostrand Reinhold, New York, NY, USA, 1974.

[29] U. Leonhardt, Measuring the Quantum State of Light, Cambridge University Press, 1997.

[30] F. Natterer, The Mathematics of Computerized Tomography, John Wiley \& Sons, Chichester, UK, 1986.

[31] T. Richter and A. Wünsche, "Determination of occupation probabilities from time-averaged position distributions," Physical Review A, vol. 53, pp. R1974-R1977, 1996.

[32] T. Richter, "Pattern functions used in tomographic reconstruction of photon statistics revisited," Physics Letters A, vol. 211, pp. 327-330, 1996.

[33] T. Richter and A. Wünsche, "Determination of quantum state from time-dependent position distributions," Acta Physica Slovaca, vol. 46, pp. 487-492, 1996.

[34] A. Wünsche, "Radon-transform and pattern functions in quantum tomography," Journal of Modern Optics, vol. 44, pp. 22932331, 1997.

[35] U. Leonhardt, "State reconstruction in quantum mechanics," Acta Physica Slovaca, vol. 46, no. 3, pp. 309-316, 1996.

[36] U. Leonhardt and M. G. Raymer, "Observation of moving wave packets reveals their quantum state," Physical Review Letters, vol. 76, no. 12, pp. 1985-1989, 1996.

[37] U. Leonhardt and S. Schneider, "State reconstruction in onedimensional quantum mechanics: the continuous spectrum," Physical Review A, vol. 56, no. 4, pp. 2549-2556, 1997.

[38] U. Leonhardt, M. Munroe, T. Kiss, T. Richter, and M. G. Raymer, "Sampling of photon statistics and density matrix using homodyne detection," Optics Communications, vol. 127, no. 1-3, pp. 144-160, 1996.

[39] U. Leonhardt, "Quantum and classical tomography with equidistant reference angles," Journal of Modern Optics, vol. 44, pp. 2271-2280, 1997.

[40] A. Messiah, Quantum Mechanics, vol. 1, North-Hollandl, Amsterdam, The Netherlads, 1965.

[41] V. A. Lobastov, J. D. Ewbank, L. Schafer, and A. A. Ischenko, "Instrumentation for time-resolved electron diffraction spanning the time domain from microseconds to picoseconds," Review of Scientific Instruments, vol. 69, no. 7, pp. 2633-2643, 1998.

[42] J. R. Pierce, Theory and Design of Electron Beams, Van-Nostrand, Princeton, NJ, USA, 1954.

[43] J. R. Helliwell and P. M. Rentzepis, Eds., Time-Resolved Diffraction, Oxford University Press, 1997.

[44] “Time-resolved electron and x-ray diffraction,” P. M. Rentzepis, Ed., vol. 2521 of Proceedings of SPIE, SPIE, Bellingham, Wa, USA, 1995.

[45] A. A. Ischenko, J. D. Ewbank, and L. Schäfer, "Structural and vibrational kinetics by time-resolved gas electron diffraction: stochastic approach to data analysis," Journal of Physical Chemistry, vol. 99, pp. 15790-15797, 1995.

[46] V. R. McCrary, R. Lu, D. Zakheim, J. A. Russell, J. B. Halpern, and W. M. Jackson, "Coaxial measurement of the translational energy distribution of CS produced in the laser photolysis of CS2 at 193 nm," The Journal of Chemical Physics, vol. 83, no. 7, pp. 3481-3490, 1985.

[47] W.-B. Tzeng, H.-M. Yin, W.-Y. Leung et al., "A 193 nm laser photofragmentation time-of-flight mass spectrometric study of CS2 and CS2 clusters," The Journal of Chemical Physics, vol. 88, no. 3, pp. 1658-1669, 1988.

[48] T. R. Todd and W. B. Olson, "The infrared spectra of ${ }^{12} \mathrm{C}^{32} \mathrm{~S}$, ${ }^{12} \mathrm{C}^{34} \mathrm{~S},{ }^{13} \mathrm{C}^{32} \mathrm{~S}$, and ${ }^{12} \mathrm{C}^{33} \mathrm{~S}$," Journal of Molecular Spectroscopy, vol. 74, pp. 190-202, 1979.

[49] I. Suzuki, "General anharmonic force constants of carbon disulfide," Bulletin of the Chemical Society of Japan, vol. 48, pp. 1685-1690, 1975.

[50] A. L. Buchachenko, "Chemistry on the border of two centuries: achievements and prospects," Russian Chemical Reviews, vol. 68 , no. 2 , p. $85,1999$. 

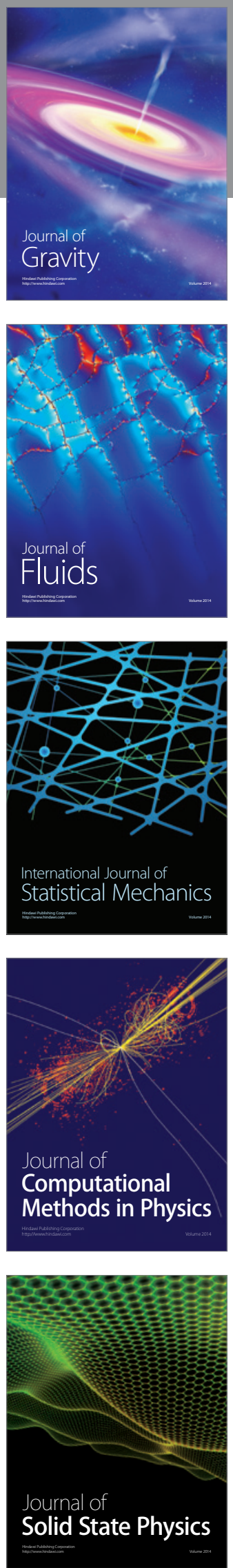

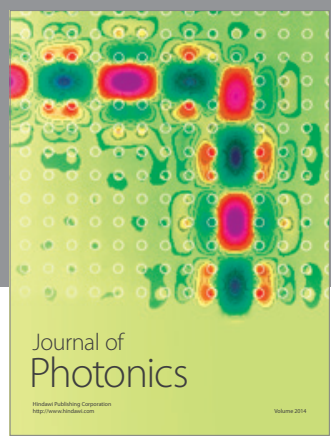

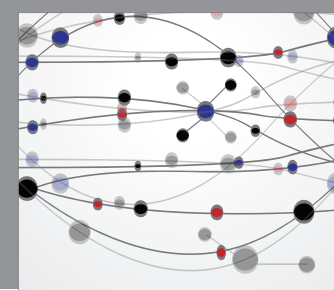

The Scientific World Journal

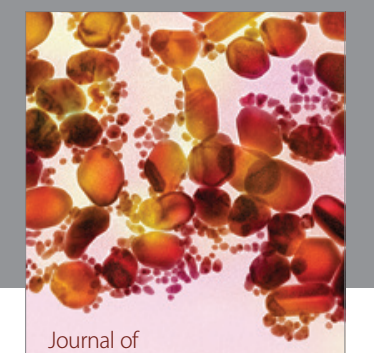

Soft Matter
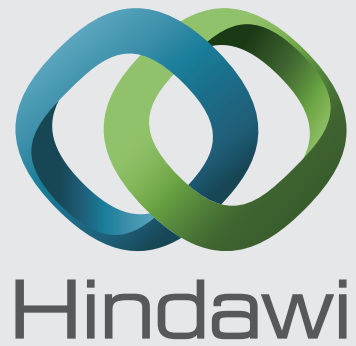

Submit your manuscripts at

http://www.hindawi.com
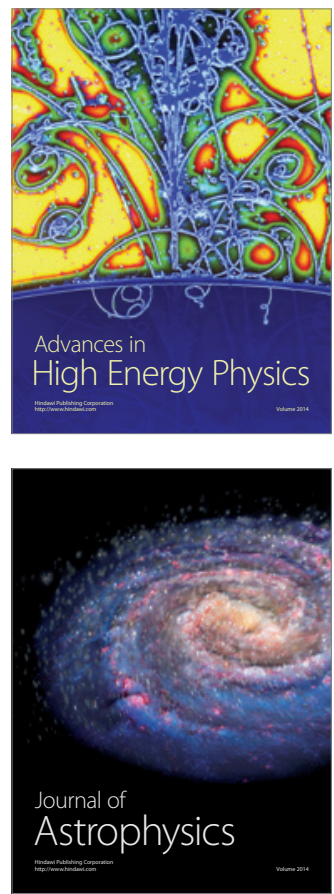
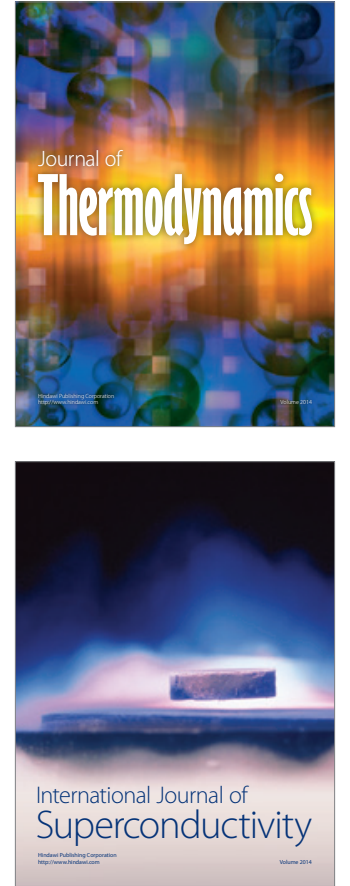
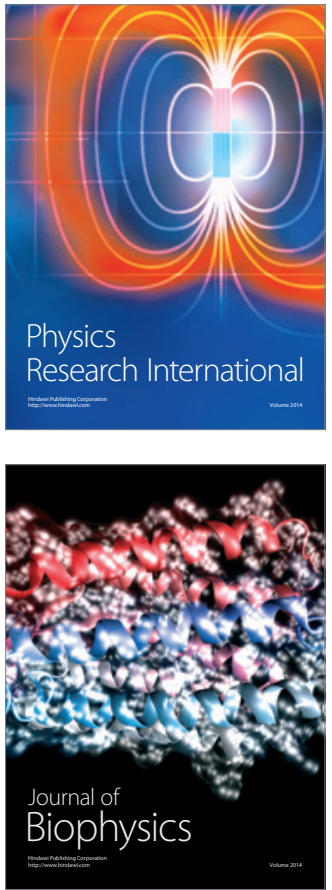
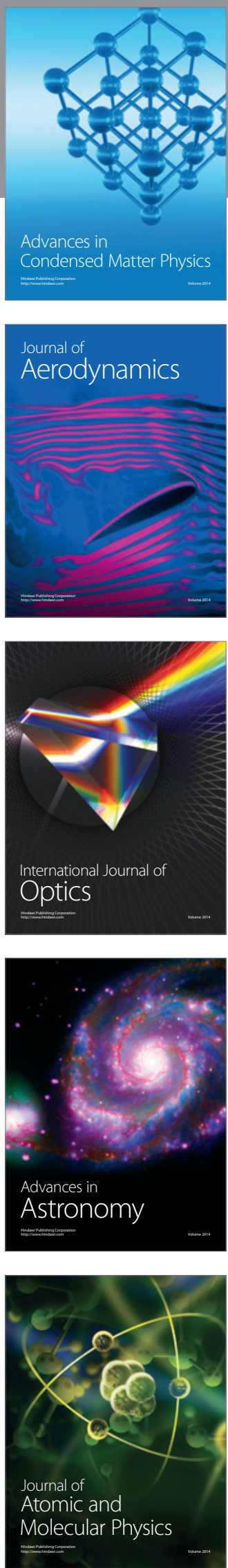